\title{
Electrical Treeing in Cable Insulation under Different HVDC Operational Conditions
}

\author{
Mehrtash Azizian Fard 1,*(D), Mohamed Emad Farrag 2,3(D, Scott McMeekin ${ }^{2}$ and Alistair Reid 4 \\ 1 School of Engineering, University of Aberdeen, Aberdeen AB24 3FX, UK \\ 2 Department of Engineering, Glasgow Caledonian University, Glasgow G4 0BA, UK; \\ mohamed.farrag@gcu.ac.uk (M.E.F.); scott.mcmeekin@gcu.ac.uk (S.M.) \\ 3 Faculty of Industrial Education, Helwan University, 11795 Helwan, Egypt \\ 4 School of Engineering, Cardiff University, Cardiff CF10 3AT, UK; reida3@cardiff.ac.uk \\ * Correspondence: m.azizianfard@abdn.ac.uk; Tel.: +44-122-427-2811
}

Received: 25 August 2018; Accepted: 7 September 2018; Published: 12 September 2018

check for updates

\begin{abstract}
Electrical treeing (ET) can irreversibly deteriorate the insulation of polymeric power cables leading to a complete failure. This paper presents the results of an experimental investigation into the effects of unipolar and polarity reversing DC voltages on electrical tree (ET) and partial discharge (PD) behavior within high voltage direct current (HVDC) cross linked polyethylene (XLPE) cable insulation. A double needle configuration was adopted to produce non-uniform electric fields within the insulation samples, potentially leading to electrical trees. The development of trees was monitored through an optical method and the associated partial discharge signals were measured through an electrical detection technique, simultaneously. The analysis of the results shows reasonable relation between the formation of ETs and the type of the applied voltages. The polarity reversing attribute of the test voltages has a pronounced effect on formation and growth of electrical trees. This implicates an interaction between the space charges that accumulate within polymeric materials and the operational polarity reversing electric fields, which causes insulation degradation. Therefore, study of influencing HVDC operational parameters on insulation degradations can contribute to improvements in cable design and advancement in insulation diagnostic strategies applicable in HVDC systems leading to more effective asset management.
\end{abstract}

Keywords: degradation; HVDC transmission; insulation; polarity reversal; partial discharges; polymers; power cable insulation; space charge; electrical trees

\section{Introduction}

HIGH voltage DC interconnections can overcome technical, economical, and operational challenges in power networks. The world's first commercial high voltage direct current (HVDC) system connecting the Gotland Island to the Swedish mainland network, via a mass impregnated (MI) cable operating at $100 \mathrm{kV}$ of $20 \mathrm{MW}$ capacity, was commissioned in 1954 [1,2]. Since then significant progress has been made in design and manufacturing technologies of power cables, electronic valves, converters, and linking configurations, which facilitate higher voltage HVDC connections and interlinks worldwide. Conventionally, polymeric cables are employed in HVDC systems operating based on voltage source converter (VSC) technology, where the interconnecting power cables are not expected to experience operational voltage polarity change for the purpose of power flow redirection. Nevertheless, incentives towards employment of more environmentally friendly insulating materials and the technological advancements are among the drives to adopt polymeric power cables in HVDC systems which are based on line commutated converter (LCC) schemes [3]. However, polymeric cables are primarily influenced by the phenomenon of space charge accumulation. The space charges are 
built up due to the interaction between DC voltages and non-regularities within the cable insulation system. The resultant electric field of these space changes and that of the operational HVDC voltage or temporary overvoltages can cause intensified degradation within the cable insulation $[4,5]$. Electrical treeing (ET) is a degradation phenomenon that deteriorates the insulation at the sites of contaminants, protrusions, or voids within the cable insulation due to locally enhanced electric fields. The irreversible progression of such deterioration which is accompanied by partial discharges leads to cable failure in the short or long term. This much depends upon the origin and the severity of the process [6,7]. Therefore, ETs are considered to be the initial stage of breakdown in power cables. Understanding the mechanism of an ET and its interaction with different electric fields under typical operational HVDC conditions would contribute to improvement in cable design and diagnostics. Different approaches have been taken so far in the electrical insulation research arena, using various electrode configurations [8-11] at DC and AC voltages to study this aging phenomenon. In addition, the effect of DC superimposed with AC voltages on electrical trees within epoxy resin has been reported in [12]. Furthermore, the effects of parameters such as temperature and magnetic fields on ETs have also been investigated in $[13,14]$.

This research work aims to address effects of operational conditions of high voltage direct current converters on PD activity and ET development within HVDC cross linked polyethylene (XLPE) cable insulation. This article has been organized as follows: in Sections 2 and 3, the preparation of test samples and the experiment setup including the experimental methodology are presented, respectively; Section 4 presents the results of PD and ET measurements under different test scenarios in association with the analyses of the results; Section 5 discusses the results and, finally, Section 6 gives the conclusion.

\section{Sample Preparation}

The double needle configuration (DNC) was adopted as the electrode system to produce non-uniform electric fields within the test samples according to ASTM standard D3756-97 [15], which could imitate a foreign object or protrusion within the main insulation of power cables. The material of the test samples was prepared from the main insulation of a $400 \mathrm{kV}$ HVDC XLPE cable.

The advantage of using actual insulation of an HVDC cable is to study the phenomenon on the material which is manufactured to be employed at HVDC conditions rather than AC cables or commonly used molded specimens of polymeric materials in research laboratories. The cable that the samples were prepared from had internal and external radii of $65 \mathrm{~mm}$ and $120 \mathrm{~mm}$, respectively. The cable was cut into discs of $3 \mathrm{~mm}$ thickness. Afterwards, the jacket of each disc was removed, and their cut-surfaces were finely polished using a grinding machine in order to allow a clearer optical observation of any prospective treeing processes. Then, the polished discs were cut into sections. Having prepared the XLPE sample, each sample was then drilled from both sides to a distance of $10 \mathrm{~mm}$ from each other. The sample preparation process is illustrated in Figure 1. The electrodes comprising a sharp needle with a tip radius of $5 \mu \mathrm{m}$ and a blunt one with the shank of $0.9 \mathrm{~mm}$ were inserted into the sample bulk. In order to reduce the mechanical stresses exerted to the insulation, the slabs were uniformly heated at $65^{\circ} \mathrm{C}$ [16] without causing any deformation then the needles were inserted into the samples in a way that the needles were arranged to be $1 \mathrm{~mm}$ distanced from each other as shown in Figure 2. To prevent any displacement the shank of needles were glued to the samples at both ends. 


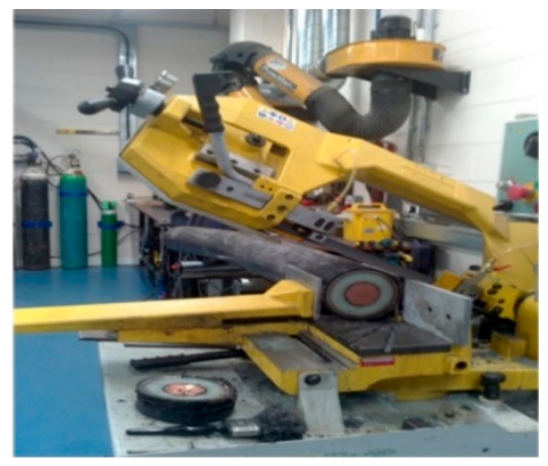

(a)

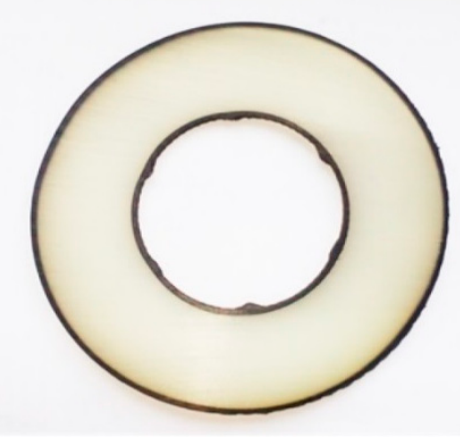

(c)

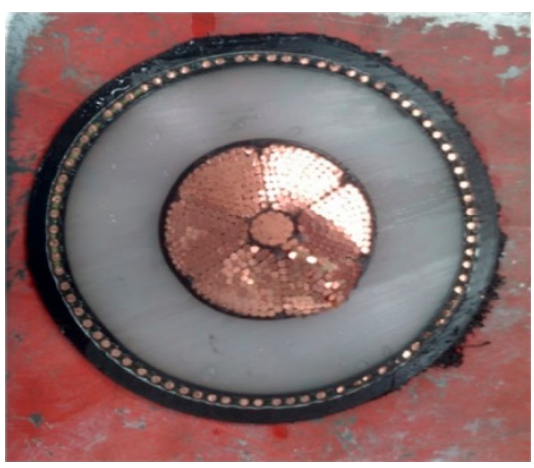

(b)

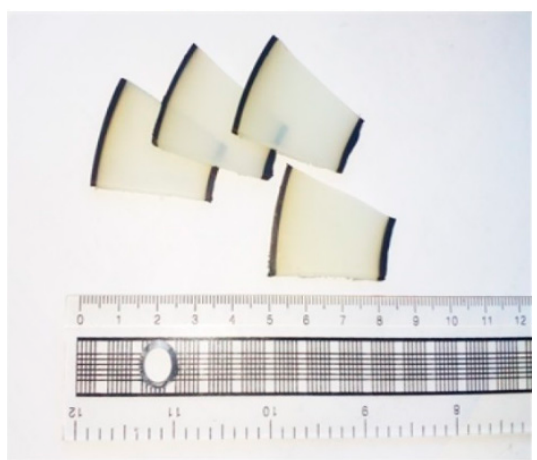

(d)

Figure 1. Test sample preparation: (a) cutting the cable into discs; (b) a disc of cable; (c) a disc without conducting part; and (d) final test samples.

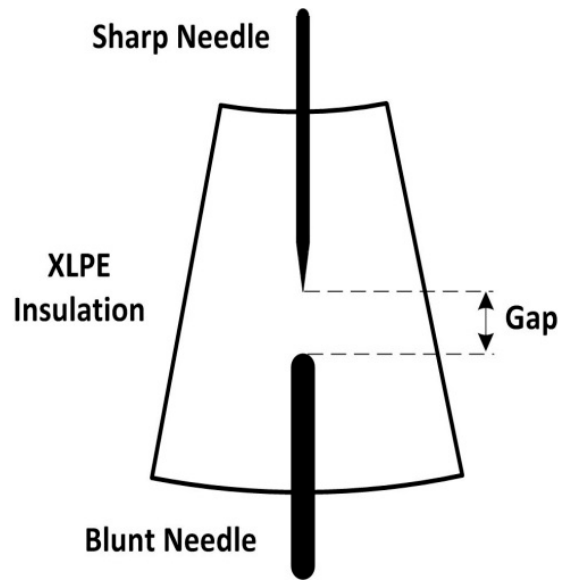

Figure 2. Electrodes arrangement in the test sample.

\section{Experiment Setup and Test Procedure}

The setup of the experiment comprises a high voltage $(\mathrm{HV})$ power source, an optical monitoring device and an electrical PD measurement system as illustrated in Figure 3. The test voltages including pure DC and operational polarity reversing voltages were produced in a programmable arbitrary signal generator. The voltage waveforms were then fed into the HV amplifier. The output terminal of the amplifier was connected to the electrode embedded within the test vessel. It is noteworthy that the ripple superimposed to the output DC voltage of the amplifier is less than $1.5 \mathrm{Vrms}$, that compared to the level of the DC test voltages is negligible. The operational gain of the $\mathrm{HV}$ amplifier is $3 \mathrm{kV} / \mathrm{V}$, which enables the amplifier to reach a maximum stable voltage of $30 \mathrm{kV}$ in both negative and positive polarities $( \pm 30 \mathrm{kV})[5]$. 


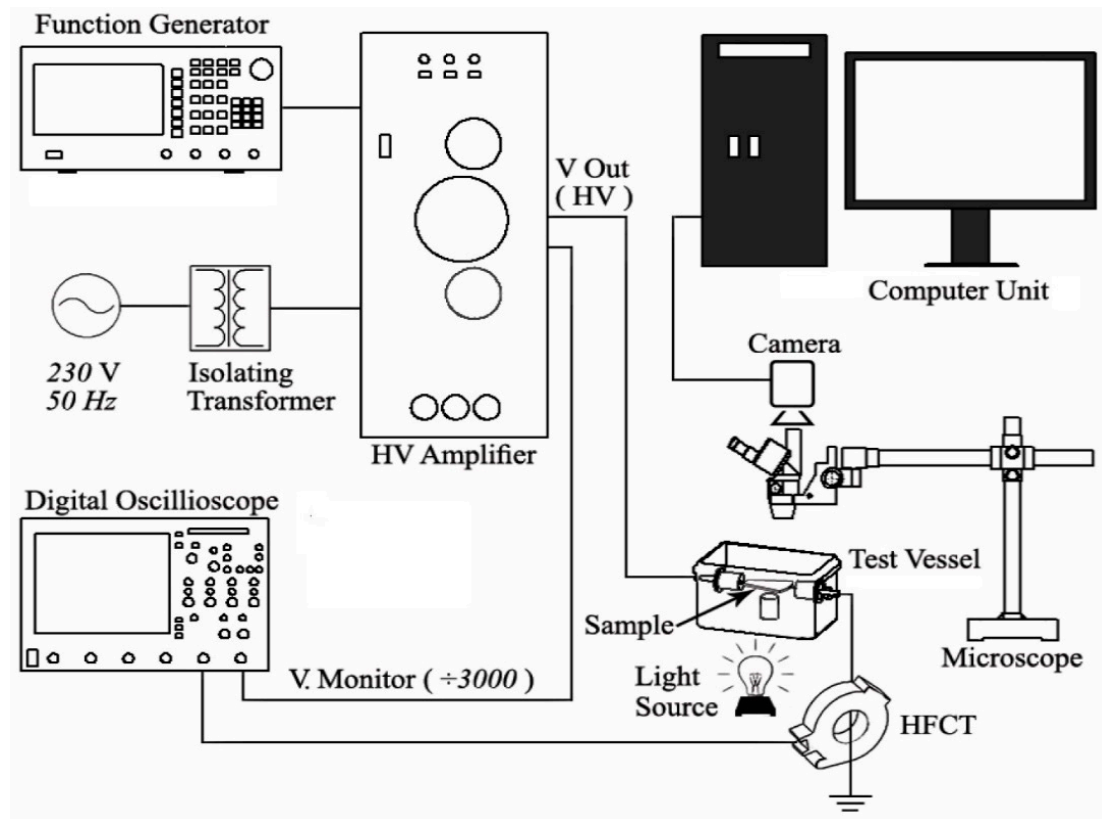

Figure 3. Experiment setup for electrical treeing (ET) optical observation and partial discharge (PD) measurement.

The prepared samples, one at a time in each experiment, were placed within the tailored test vessel. The sample within the vessel was immersed in transformer oil to prevent possible corona discharges or flashover. In addition, the transformer oil helps in increasing the resolving power of the microscope due to its high refractive index $[17,18]$. The microscope was arranged in a direction towards the test sample, as shown in the schematic, with its objective lens in a proper clearance from the test sample. The camera was attached to the microscope through its phototube and was connected to a desktop computer to record images of the process of prospective treeing within the samples for further analysis. The camera is of CMEX model, CMOS sensor technology, which has a maximum resolution of $2592 \times 1944$ pixels, and it has the sensitivity of $1.3 \mathrm{~V} /$ lux-s.

Simultaneously, the associated PD pulses of treeing process within the samples were detected using a high frequency current transformer (HFCT). The HFCT was suitably clamped around the ground wire of the test vessel. The sensitivity of the HFCT is $4.3 \mathrm{mV} / \mathrm{mA} \pm 5 \%$ within a wideband frequency detection range from $0.2 \mathrm{MHz}$ to $19 \mathrm{MHz}$. The output terminal of the HFCT was appropriately connected to a digital oscilloscope (Lecroy) using a coaxial cable.

\section{Experimental Results}

\subsection{Electrical Treeing under Pure HVDC}

In the scenarios of pure DC voltages, the phenomenon of treeing was investigated at continuous and non-continuous DC voltages as proposed in [19], where treeing phenomenon was investigated using needle-plane electrode arrangement. The first scenario can be considered as the long-time operation of an HVDC link in either polarity; DC voltages of linearly increasing function were applied to the test sample, in a way the voltage increased from zero level with the rising rate of $500 \mathrm{~V} / \mathrm{s}$ to a predetermined value. During the voltage subjection time of $2 \mathrm{~h}$, the initiation of treeing and its propagation was monitored through a real-time optical observation, and electrical PD detection. The PD signals were monitored with a pre-determined trigger level applied to suppress the effects of surrounding background noise. Three different voltage levels were applied in these test series, where each series included ten samples. The first voltage level was selected according to Equation (1) which has been proposed in [20] for the calculation of electric field in a double electrode configuration and the reported treeing inception strength within XLPE material in [21]. The maximum 
Laplacian electric field stress-without considering the effect of space charges—can be calculated using Equation (1):

$$
\mathrm{E}_{\max }=\frac{2 U}{r \ln \left[\frac{2 d}{r}\left(1+\frac{2 d}{R}\right)\right]},
$$

where $U$ is the potential difference across the needle electrodes, $d$ is the gap distance between electrodes, $r$ and $R$ are the respective radii of the sharp and the blunt needles. The second and the third voltage levels were attained through increasing the first voltage in subsequent increments of $3 \mathrm{kV}$. The results of tree formation under these three voltage levels are given in Table 1.

Table 1. Tree inception at continuous DC voltages.

\begin{tabular}{ccc}
\hline \multirow{2}{*}{ Voltage Level (kV) } & Positive DC & Negative DC \\
\cline { 2 - 3 } & $\begin{array}{c}\text { Percentage of Samples in Which } \\
\text { ET's Initiated (\%) }\end{array}$ & $\begin{array}{c}\text { Percentage of Samples in Which } \\
\text { ET's Initiated (\%) }\end{array}$ \\
\hline 22.0 & 0 & 0 \\
25.0 & 20 & 0 \\
28.0 & 20 & 0 \\
\hline
\end{tabular}

In the second scenario, the same three DC voltage levels of the same rising rate but in non-continuous form were applied to another set of similar test samples, where after the time duration of $10 \mathrm{~min}$ the test voltage lowered to ground level. Table 2 shows the observational results of ET initiation at non-continuous DC voltages, for both positive and negative polarities.

Table 2. Tree inception at non-continuous DC voltages.

\begin{tabular}{ccc}
\hline \multirow{2}{*}{ Voltage Level (kV) } & Positive DC & Negative DC \\
\cline { 2 - 3 } & $\begin{array}{c}\text { Percentage of Samples in Which } \\
\text { ET's Initiated (\%) }\end{array}$ & $\begin{array}{c}\text { Percentage of Samples in Which } \\
\text { ET's Initiated (\%) }\end{array}$ \\
\hline 22.0 & 0 & 0 \\
25.0 & 20 & 20 \\
28.0 & 50 & 60 \\
\hline
\end{tabular}

Comparing the ET initiations at continuous and non-continuous scenarios, as given in Tables 1 and 2, the number of test samples leading to ET initiations is higher in the case of non-continuous DC voltages. Furthermore, the frequency of ET initiations at pre-stressed negative DC voltage is higher with respect to the ones at pre-stressed positive DC voltages. It can be deduced that the treeing initiation depends on the electric field strength and the polarity. In the second scenario, the grounding after each pre-stress forms a fast-transient electric field due to already accumulated space charges. The effect of such abrupt electric field change could be of enough energy to locally degrade the insulation and, hence, leads to formation of tree channels [22]. Particularly, the experimental results, as given in Table 2, suggest that ET resistivity of the test samples at negative DC is lower than positive DC voltages while it is the opposite case in the first scenario. This could be due to the fact that injection and de-trapping of negative charge carriers are easier compared to their positive counterparts [23].

Furthermore, the experimental results show that ET initiation voltages of non-continuous DC are lower than those of the continuous DC voltages. This might be due to the effect of the grounding mechanism through which the already built up space charges and the polarized bounded charges redistribute within the geometry as a result of the grounding effect. As it can be deduced from the treeing voltage levels, under different voltage polarities, the accumulated space charges are of homo-charges. Depending on the test voltage level, and the material of test sample, the depth of trapped space charges varies [11]. When the sharp needle is grounded, an electric field will form due to potential difference between the space charges and the grounded needle. The higher the charge level, 
the more intense will be the electric field strength. Such an electric field could provide the condition for partial breakdown of the insulation between the region of space charges and the electrode, which leads to the formation of passages from the accumulation area of the space charges to the ground. This, in turn, helps forming tree channels [24]. Figure 4a,b depict the development of ETs at positive and negative voltages, respectively.

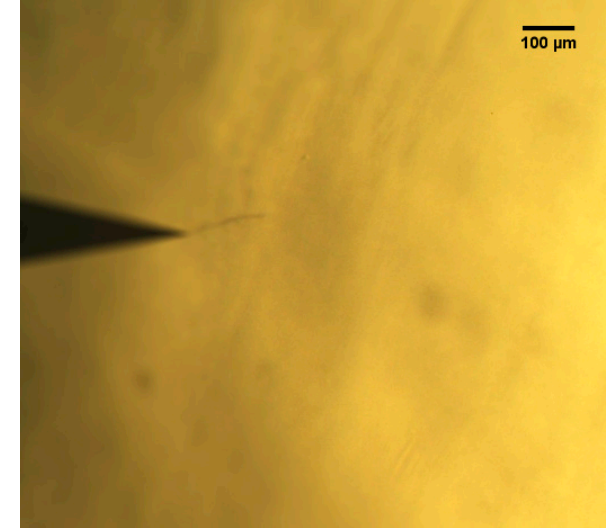

(a)

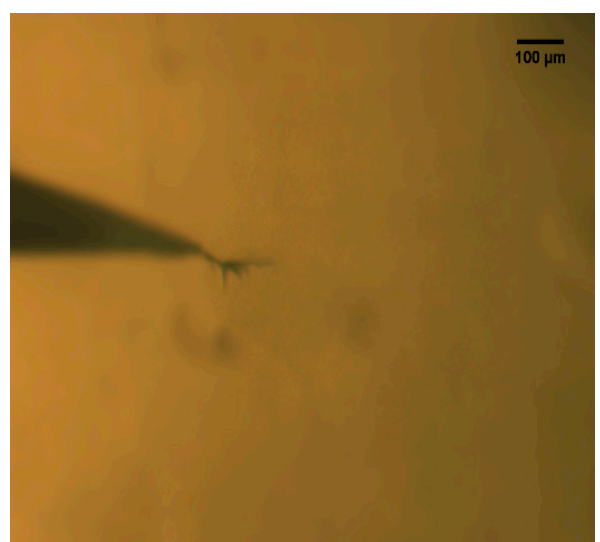

(b)

Figure 4. ETs developed at DC voltage (a) $+28 \mathrm{kV}$ and $(\mathbf{b})-28 \mathrm{kV}$.

\subsection{Electrical Treeing under Polarity Reversing HVDC}

Polarity reversals in LCC HVDC systems are applied depending upon the purpose of the linkage. For example, polarity changing is employed to redirect the power flow to the point where it is needed to meet the market demands, which could be per day, month, or longer time spans. Generally, depending on the situations, two types of polarity reversal are applied in LCC HVDC links: fast and slow [6]. In the fast polarity reversing, the time duration that elapses to change from one polarity to another is about one second. Here, the effects of polarity reversals on treeing were investigated without the consideration of the superimposed harmonics in LCC systems [2]. Test voltages of the same levels, as in the former scenarios, but in bipolar manner were applied to the test samples as given in Figure 5, where the ET developments were monitored.

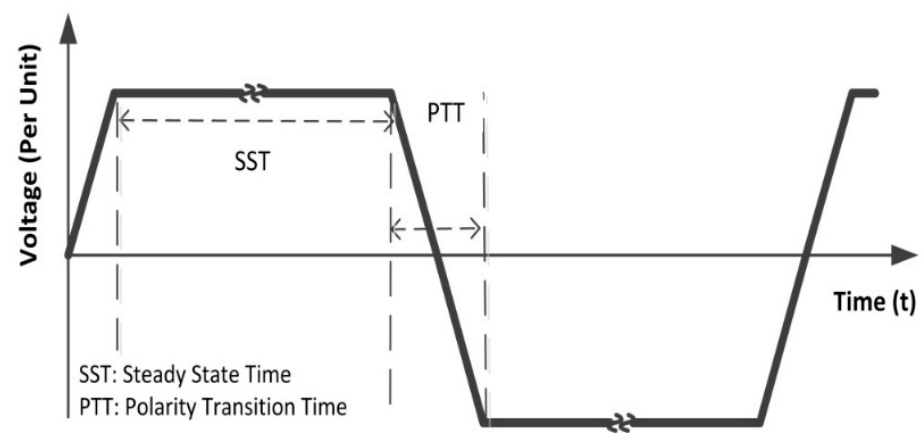

Figure 5. A typical applied polarity reversing voltage.

The effects of voltage level and the polarity transition time on ET development were investigated at three different voltages with three different transition times, and Table 3 shows the results of the experiments. In comparison with the results obtained in the unipolar DC voltage scenarios, the percentage of the samples in which ETs initiated is higher, while the ET initiation voltage is lower with respect to the previous cases. This might be due to the effect of space charges and their interaction with the externally applied voltages. At the instant of polarity changing, the abrupt change in the voltage in synergy with the electric field produced by the already accumulated space charges leads to 
an enhanced localized electric field capable of initiating PDs. Such partial discharge activities can then lead to further insulation degradation and, hence, ET development [7].

Table 3. Tree initiations at polarity reversing voltages.

\begin{tabular}{cccc}
\hline & \multicolumn{3}{c}{ Polarity Transition Time (s) } \\
\cline { 2 - 4 } Voltage Level (kV) & $\mathbf{1}$ & $\mathbf{1 0}$ & $\mathbf{1 0 0}$ \\
\cline { 2 - 4 } & \multicolumn{3}{c}{ Percentage of Samples in Which ET's Initiated (\%) } \\
\hline 22.0 & 40 & 10 & 10 \\
25.0 & 70 & 60 & 30 \\
28.0 & 90 & 70 & 80 \\
\hline
\end{tabular}

As the duration of polarity transition time increases the tree initiation voltage increases leading, generally, to a lower percentage of ET initiation. This might be due to the dynamic effect of voltage gradient on the already accumulated space charges, where under the faster polarity changing the moderation effect of homo-charges are less effective than that of the slower transitions. This factor in association with higher applied voltages produce an enhanced electric field to cause local breakdown and degradation of the insulation leading to tree formations. Figure 6a-d illustrate a typical ET initiation, development, growth, and final breakdown under the polarity reversal scenario.

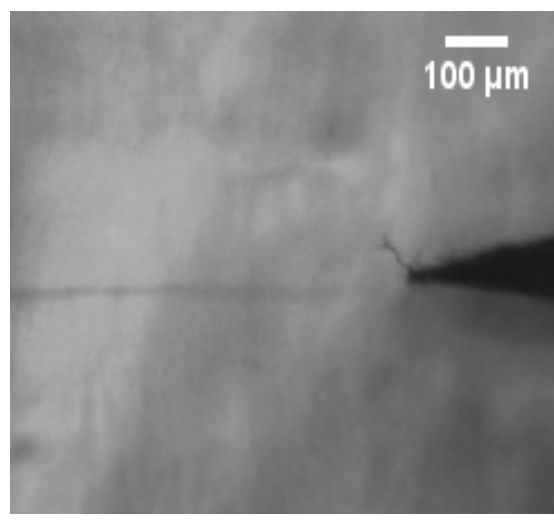

(a)

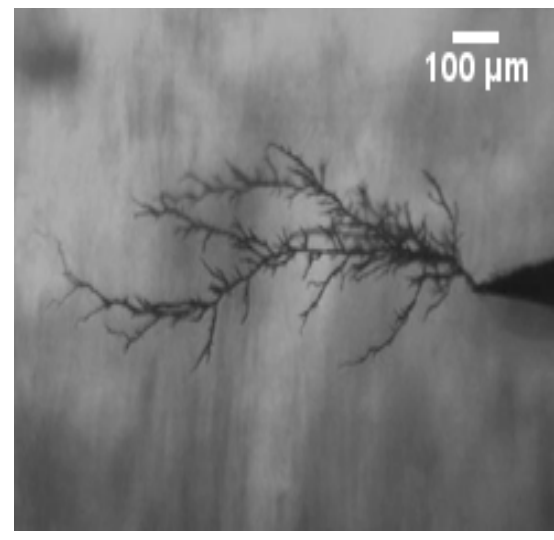

(c)

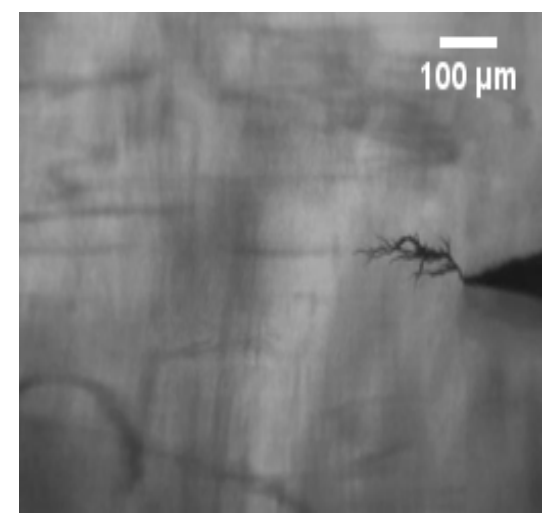

(b)

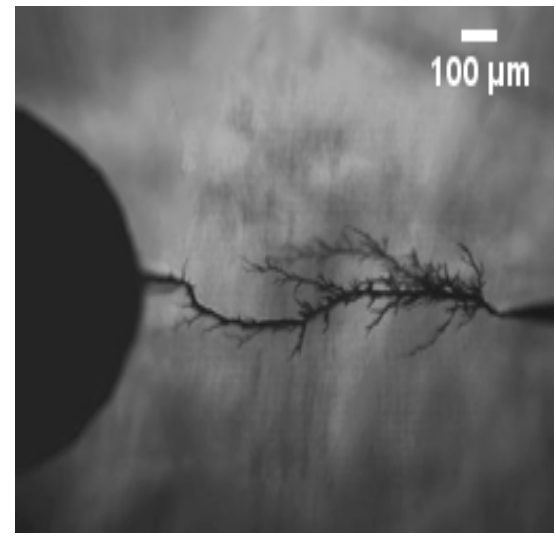

(d)

Figure 6. ET: (a) initiation; (b) development; (c) propagation; and (d) breakdown at polarity reversal of $28 \mathrm{kV}$.

In order to investigate the effects of steady state and polarity transition time of test voltages on electrical tree propagation, four different cases, as given in Table 4, have been considered, and Figure 7 shows the mean tree length of ET propagations in the course for these cases. 
Table 4. Characteristics of the applied polarity reversing voltages.

\begin{tabular}{ccc}
\hline Case & SST (min) & PTT (s) \\
\hline I & 1 & 1 \\
II & 5 & 1 \\
III & 5 & 10 \\
IV & 5 & 100 \\
\hline
\end{tabular}

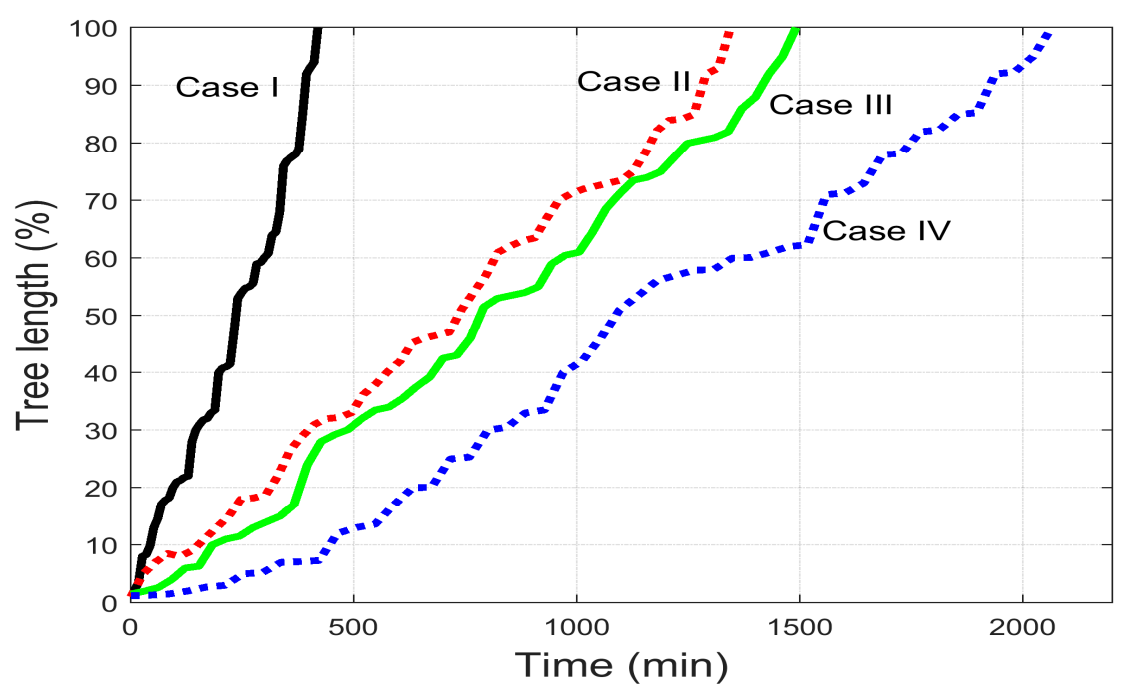

Figure 7. Typical tree growth characteristics for four polarity reversals.

The graph indicates that polarity transition and the unipolar voltage subjection time influence the ET propagation rate due to the dynamic of the applied voltage. A considerable difference in growth rates is observable under different steady state time (SST) and polarity transition time (PTT), where case (I) has faster tree propagation than the other cases. As in case (I) the unipolar time is less than others, so the amount of space charges that can build up and be trapped in the depth of the insulation is lower and, hence, has less field modification influence as opposed to cases (III)-(IV). As the polarity transition time decreases the propagation rate increases. This can be explained that in the case of fast polarity transitions the already accumulated space charges redistribute faster and with the effect of the externally applied voltage it causes a transient enhanced electric field leading to more PD activity and hence higher rate of ET growth.

Furthermore, it was observed that the structural shapes of the formed ETs were influenced by the type of the applied voltages. The trees that formed at polarity reversal scenarios compared to those formed at steady DC voltage have wider structures with more minor and fine branches. In addition, the structure of trees that formed at higher voltages has wider dimensions than those formed at lower voltages. Such characteristics of geometrical structures of ETs were investigated using fractal dimension $[11,25,26]$. The recorded images of ETs were converted into binary format in MATLAB (R2017b, MathWorks, Natick, MA, USA) and using the box counting method [27] the fractal dimensions were extracted for each case. Figure 8 shows the fractal dimension of the ETs as a function of polarity transition time. The graph indicates that as the transition time of the test voltage increases the fractal dimension of the ETs decreases, which is a quantitative index of the geometrical complexity of the tree structures. At the subjection of voltages with fast transition, the number of tree channels is greater than that at the slower transition time. This is due to the effect of more abrupt electric fields appearing under the former, leading to a higher PD activity and more degradation and, therefore, more branches. It is noteworthy that growing structure of the trees might be restricted to the sides of the samples or stagnated before reaching the sides due to material morphology and electrical field intensity reduction in these regions (top and bottom sides according to the test configuration in Figure 3). 


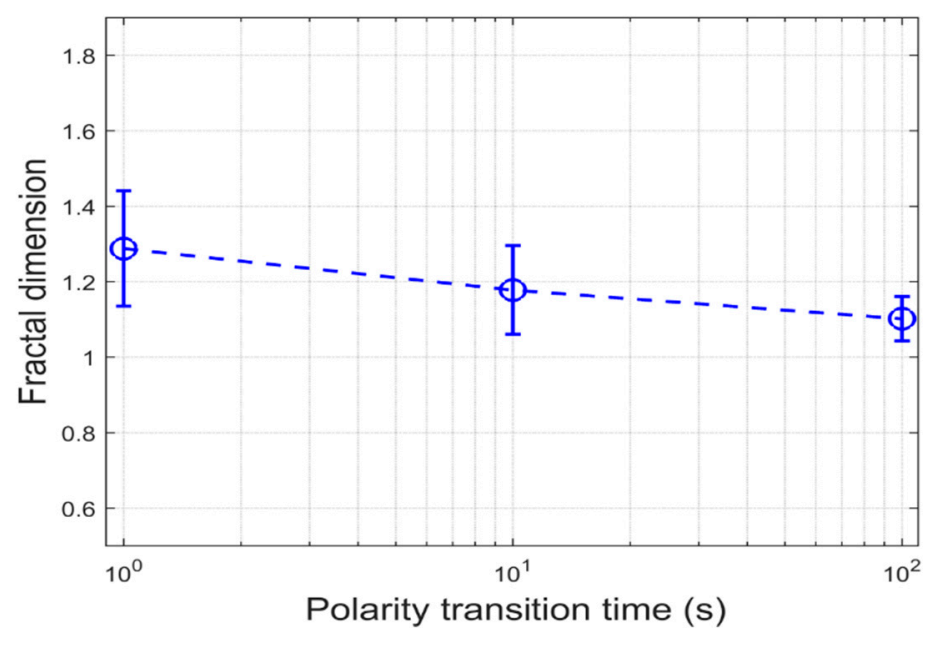

Figure 8. Fractal dimension as a function of polarity transition time.

Figure 9 illustrates fractal dimensions for the ETs that formed under three different scenarios of polarity reversals. All the test voltages have the same magnitude of $28 \mathrm{kV}$ and PTT of $1 \mathrm{~s}$, but their SST varies as 1,5, and $10 \mathrm{~min}$. As the graph indicates, fractal dimensions are influenced by the duration of DC steady state time, suggesting that the trees developed under periodic polarity reversal of shorter SST have higher number of branches. However, the ETs formed under the test voltage of longer SST have approximately similar values of fractal dimensions, i.e., for SST of 5 and $10 \mathrm{~min}$. This situation could suggest that for SST longer than the threshold, the accumulation of space charges reaches its saturation level. After each polarity changing under the fast transition and short steady state DC, the number of flake and minor channels that stemmed from the main channels is higher than the cases of slow polarity transitions and longer steady state DC. This might be due to the effect of boosted electric field that happens under the former cases. However, under the slow transition, mainly the existing channels extend with fewer new minor channels.

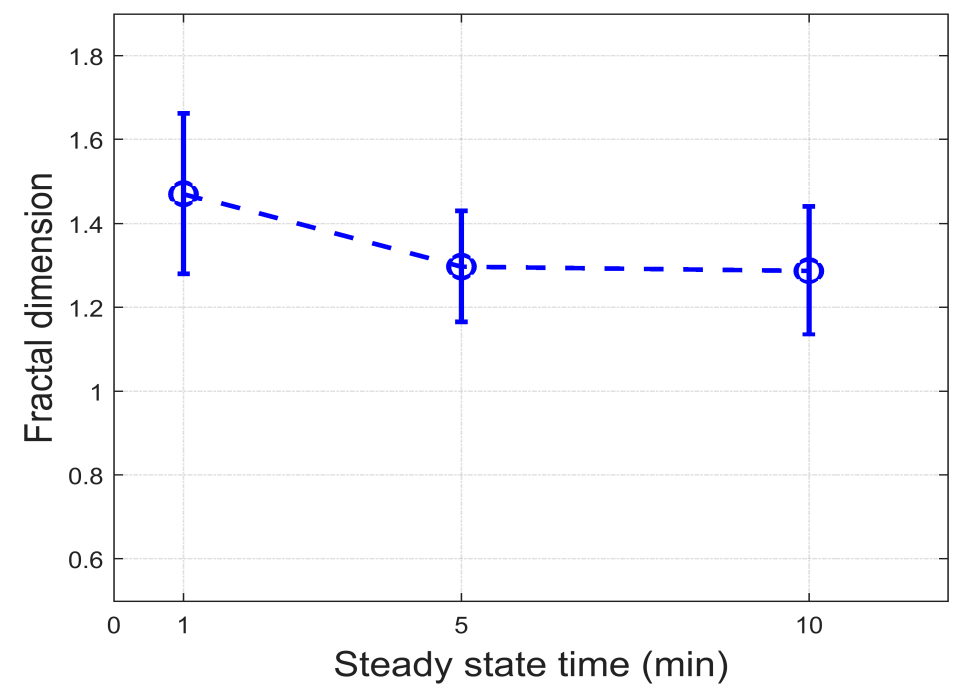

Figure 9. Fractal dimension as a function of steady state time.

\subsection{PD Behavior at Different Stages of ET Growth}

The PD activity was monitored during each experiment. There were rarely detectable PD activities observed until the initiation phase of ETs. However, as the first sprouts of treeing appeared, associated PD pulses were detected. Figures 10 and 11 illustrate the PD activities during the transition time from negative to positive polarity and vice versa, respectively. 


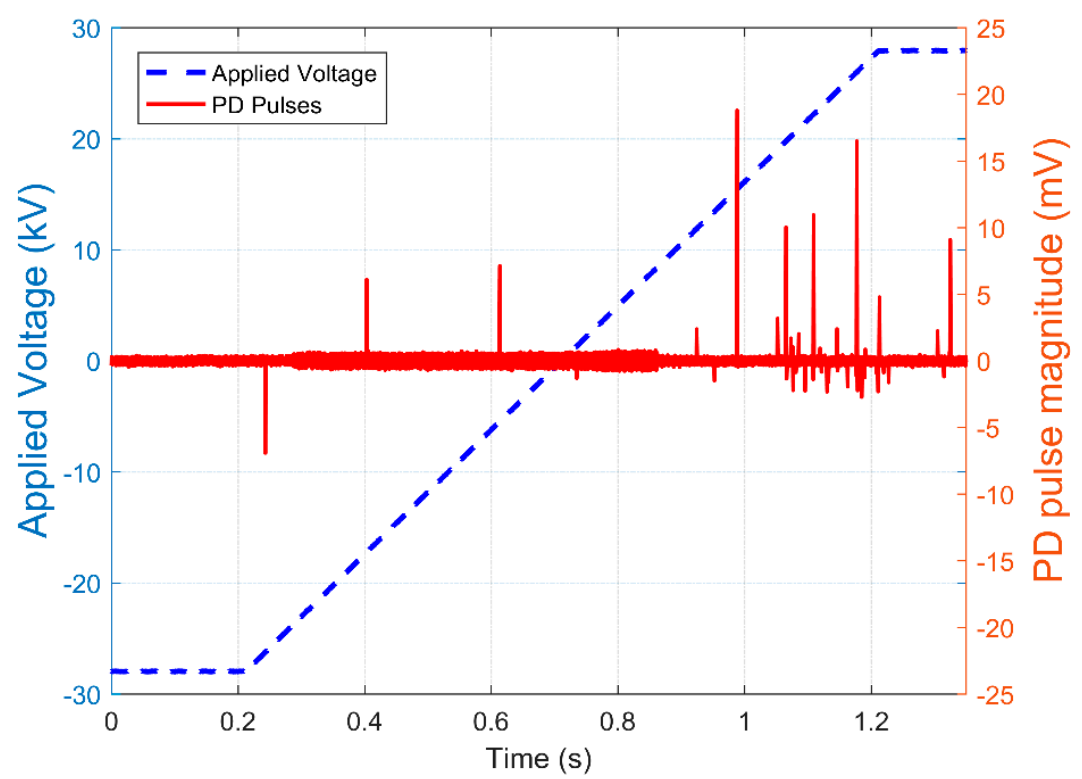

Figure 10. Partial discharge during the polarity change from negative to positive $(28 \mathrm{kV})$.

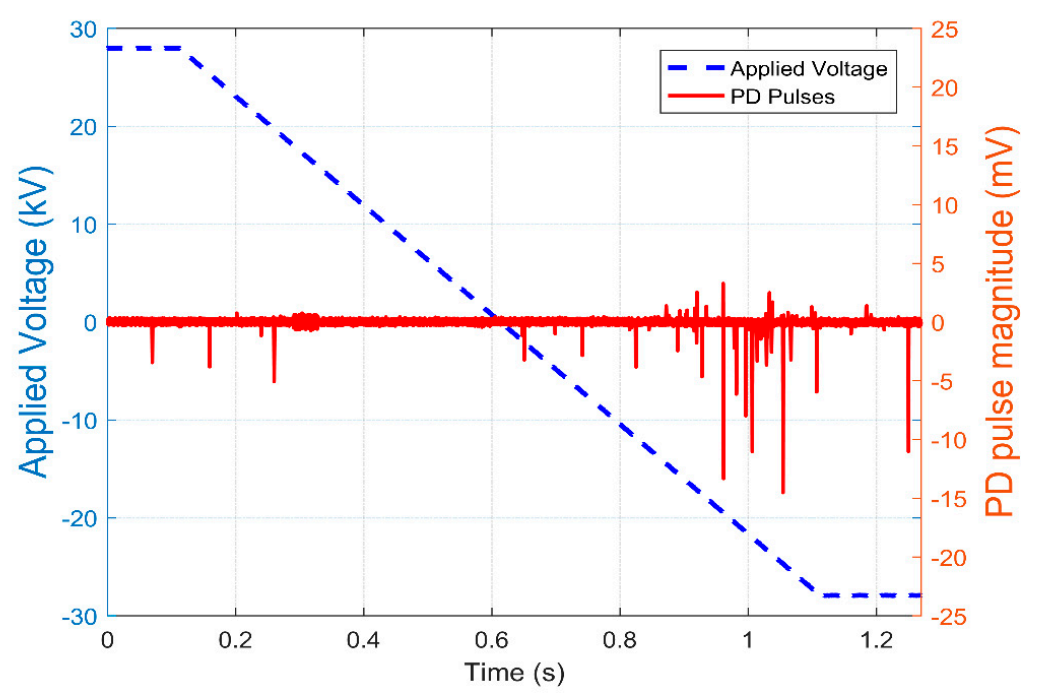

Figure 11. Partial discharge during the polarity change from positive to negative $(28 \mathrm{kV})$.

Figures 12-15 depict the ETs along with their PD activities in the course of their growth for cases aforementioned in Table 4. There are two major PD characteristics that are common in all the scenarios: firstly, the PD activities have a changing dynamic with burst-like increase in PD activity. These bursts generally correspond to the points at which ETs have a higher growth rate. Such behaviors might be explained as a result of the total damages that occur due to the large number of discharges that lead to more degradation and, therefore, the extension of ETs. Secondly, PD rates have an increasing trend in the course of time. As a tree extends towards the counter electrode, the damage that the PDs leave behind within the insulation form the branches and flakes of the tree. These channels are either conductive or nonconductive. In the conductive branches, the tips of the channels perform as extensions to the needle and the electric fields at these tips are higher compared to the original needle tip, this could increase the likelihood of PD occurrence. On the other hand, within the branches of non-conducting channels there is the possibility of gas discharge providing the discharge conditions are fulfilled [11]. The electrical method of PD detection using HFCT sensor, adopted here, is in good consistency with the optical method that has been proposed in [28], where the PD signals are detected using a silicon multiplier (SiPM) sensor, which is promising technique in suppression of electromagnetic (EM) interference and effective PD acquisition. 


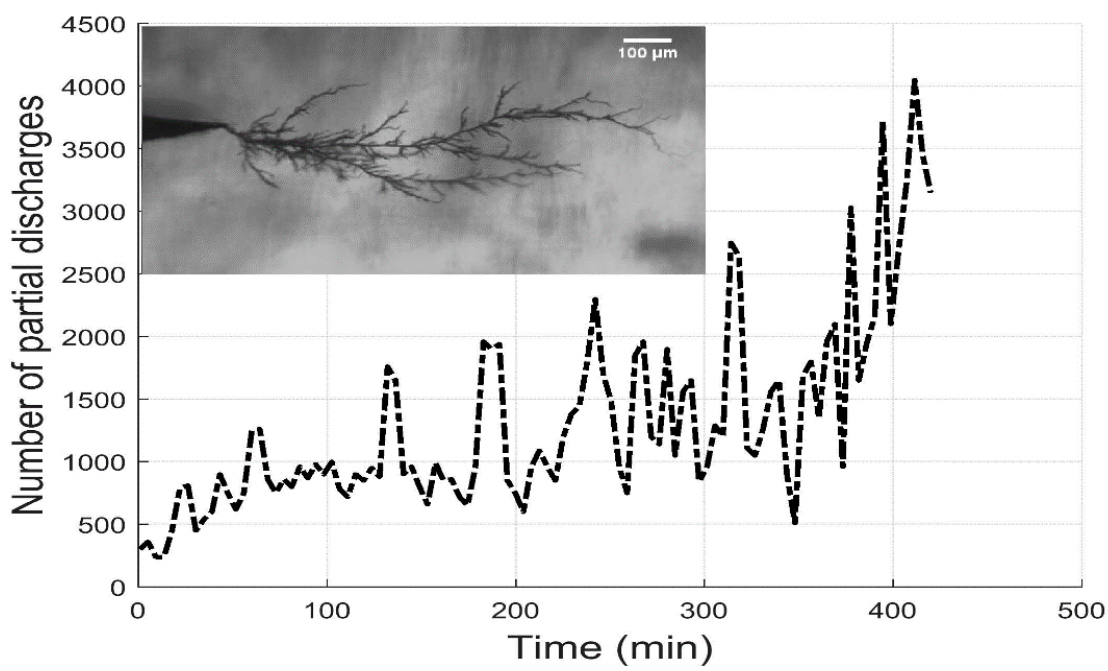

Figure 12. Partial discharge activities of ET growth under polarity reversal for case (I) in Table 4.

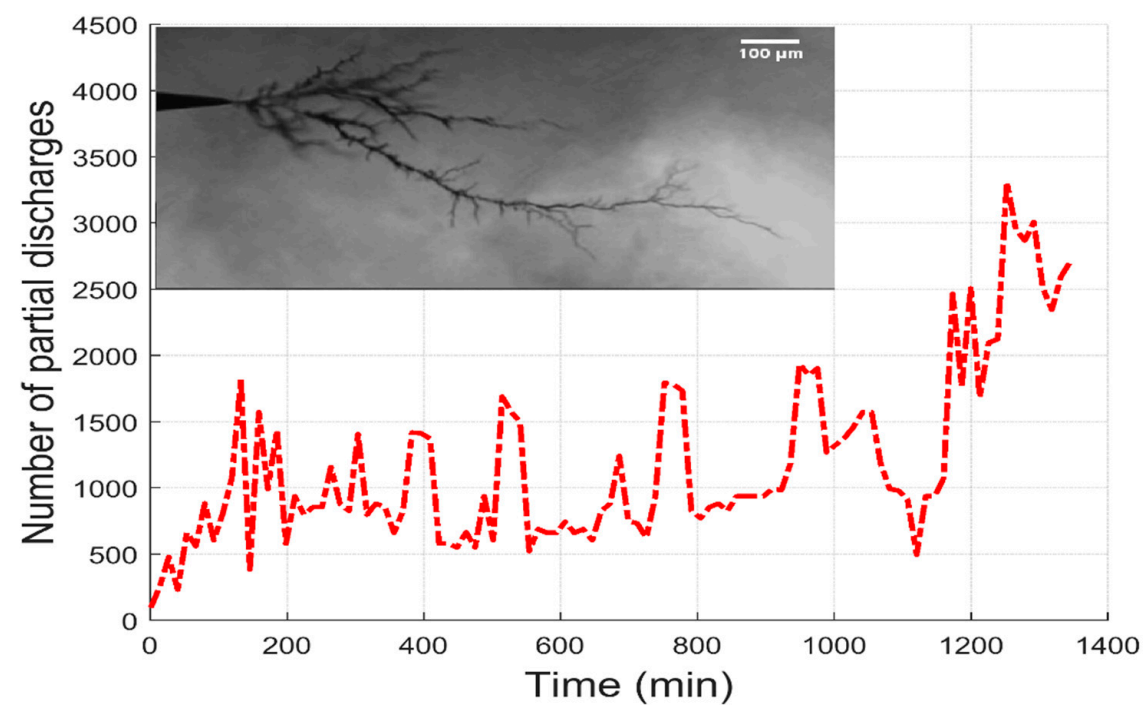

Figure 13. Partial discharge activities of ET growth under polarity reversal for case (II) in Table 4.

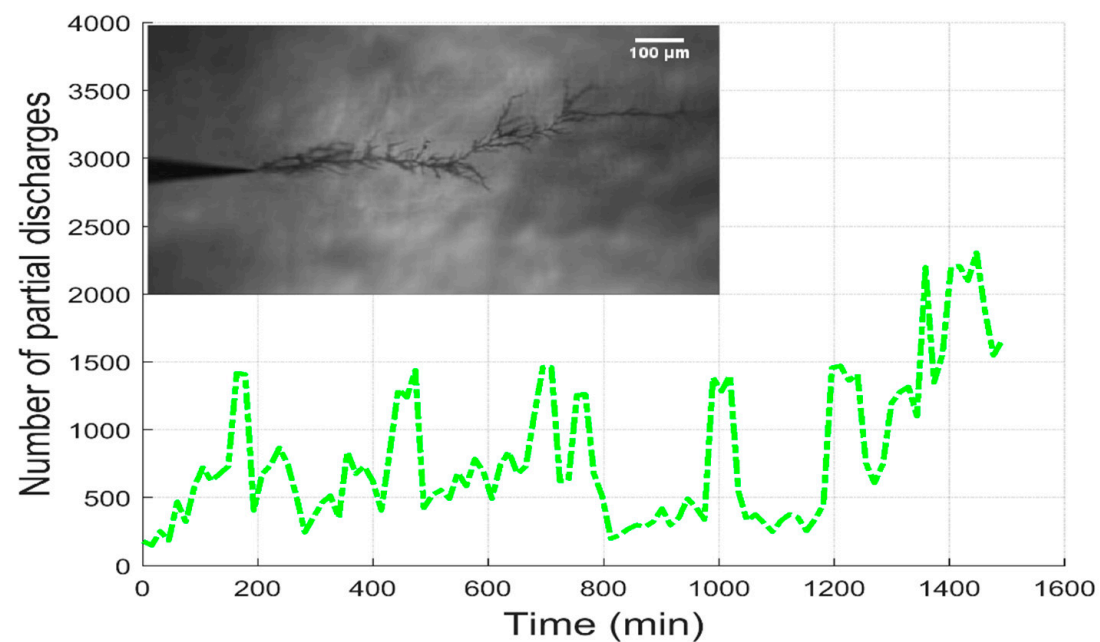

Figure 14. Partial discharge activities of ET growth under polarity reversal for case (III) in Table 4. 


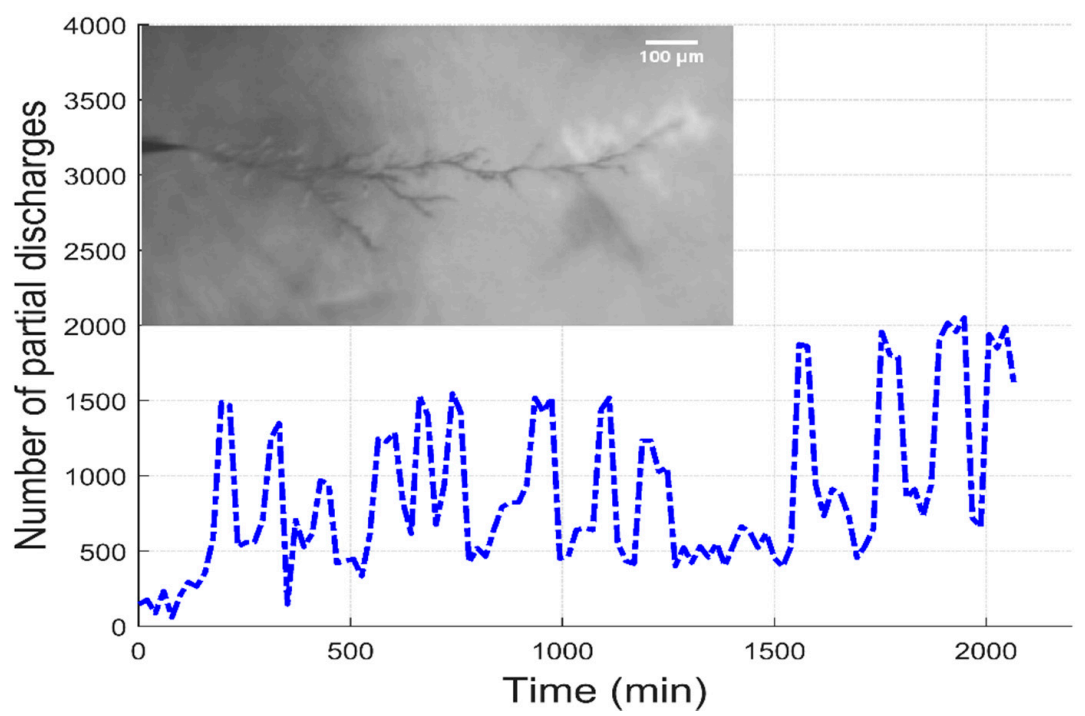

Figure 15. Partial discharge activities of ET growth under polarity reversal for case (IV) in Table 4.

\section{Discussion}

In general, the experimental results indicate that electrical trees are influenced by the type of voltage and method of application. ETs and the associated PDs have higher activities in terms of extension length and frequency of occurrences at the scenario of polarity reversal compared to the pure unidirectional DC voltages. This might be explained thusly due to de-trapping and redistribution effects of the already accumulated space charges. When subjected to dynamic fields at polarity reversal they contribute to such phenomenal behaviors. Electrical trees initiated at higher magnitude of negative DC voltages compared to positive polarity voltages, which is due to the faster mobility of negative charge carriers and space charges building up at the vicinity of non-homogeneities within the insulation bulk and interfaces. This feature has a modification influence on the electric field at the tip of the needle which decreases the electric field leading to inception of trees at higher negative DC voltage.

Under DC voltages, the treeing shape is a specific type of branch in which the structure comprises one or two main branches (channels) and a few minor branches that originate from the major branches (channels). In the course of aging and degradation, one of these major branches leads the treeing process towards the counter electrode. The number of channels that developed at negative polarity is more than those at positive polarity, and furthermore the growth rate of channels at negative voltage is higher, particularly in the scenario of non-continuous DC voltage. However, under polarity reversal, generally, the treeing shapes are of crowded branches. In which the number of major branches (channels) are higher. The density of crowded branches depends on the magnitude of the voltage, steady state time of each polarity and the polarity transition time. As the steady state time and the polarity transition time decrease, the level of inter-linkage between the channels increases as does number of the small minor branches, hence increasing the fractal dimension of the tree structure.

Under the scenario of negative pre-stressing DC and polarity reversal, it was observed that the PD activities are generally higher than the PD at pre-stressed positive voltage, and as the ETs progress towards the counter electrode this activity increases with higher activity of PD pulses, which is in agreement with the higher growth rate of branch lengths that were observed visually.

Generally, the treeing process unanimously observed within the test samples comprises three stages; the first stage is from the application time of test voltage until the appearance of observable tree. This stage is mostly the longest stage in terms of time. The second stage is called the propagation stage, during this stage the initiated tree grows and, in the course of time, new major and minor channels and branches form. Lastly, the final stage occurs during which the already approached channels 
or branches to the counter electrode develop the bridging connections leading to insulation failure. This stage, in comparison to the former stages, is the shortest one observed during the experiments.

\section{Conclusions}

The effects of DC voltages, in the form of unidirectional and polarity reversal, on ET inception and growth along with PD activity within DC XLPE insulation were investigated. It was shown that the structure of trees and activities of the accompanied PD pulses generally depend upon the magnitude and dynamic characteristics of the applied voltages. The probability of ET occurrence is higher in the case of polarity changing due to synergic effect of accumulated space charges. This shows a field-dynamic dependent degradation at the site of insulation anomalies (e.g., metallic contaminant in this investigation). Furthermore, there is a reasonable correlation between ET progression and its accompanied PD activity.

As electrical tree is one of the main causes of power cable failure, understating the mechanism of such a degrading phenomenon under operational conditions of HVDC and its interrelation with the concurrently acquired partial discharge signals could provide valuable data for network operators in assessing the insulation condition of critical assets such as sub-sea and other cable interlinks. This could potentially facilitate pre-empting of catastrophic insulation failures by integration of ET severity with online PD monitoring data in these asset classes.

Author Contributions: Methodology, M.A.F., M.E.F., and A.R.; Investigation, M.A.F.; Writing-Original Draft Preparation, M.A.F.; Writing-Review \& Editing, all the authors.

Funding: This research received no external funding.

Conflicts of Interest: The authors declare no conflict of interest.

\section{References}

1. Special Report: 60 Years of HVDC. Available online: https:/ / new.abb.com/motors-generators/about-us/ document-library (accessed on 20 December 2014).

2. Arrillaga, J. High Voltage Direct Current Transmission, 1st ed.; Institute of Electrical Engineering: London, UK, 1998; ISBN 13: 9780852969410.

3. Murata, Y.; Sakamaki, M.; Abe, K.; Inoue, Y.; Mashio, S.; Kashiyama, S.; Matsunaga, O.; Igi, T.; Watanabe, M.; Asai, S.; et al. Development of high voltage dc-Xlpe cable system. SEI Tech. Rev. 2013, 101, 55-62.

4. Morshuis, P.; Cavallini, A.; Fabiani, D.; Montanari, G.C.; Azcarraga, C. Stress conditions in HVDC equipment and routes to in service failure. IEEE Trans. Dielectr. Electr. Insul. 2015, 22, 81-91. [CrossRef]

5. Azizian Fard, M.; Reid, A.J.; Hepburn, D.M.; Farrag, M.E. Partial discharge behavior under HVDC superimposed with transients. In Proceedings of the 51st International Universities Power Engineering Conference (UPEC), Coimbra, Portugal, 6-9 September 2016; pp. 1-5.

6. Marzzanti, G.; Marzinotto, M. Extruded Cables for High Voltage Direct Current Transmission: Advance in Research and Development; Wiley-IEEE Press: Hoboken, NJ, USA, 2013; ISBN 978-1-118-09666-6.

7. Montanari, G.C.; Dissado, L.A.; Serra, S. The hidden threat to HVDC polymeric insulation at design field: Solitonic conduction. IEEE Electr. Insul. Mag. 2014, 30, 39-50. [CrossRef]

8. Dissado, L.A.; Fothergill, J.C. Electrical Degradation and Breakdown in Polymers, 1st ed.; Peter Peregrinus Ltd.: London, UK, 1992; ISBN (0863411967).

9. Schurch, R.; Rowland, S.M.; Bradley, R.S.; Withers, P.J. Imaging and analysis techniques for electrical trees using X-ray computed tomography. IEEE Trans. Dielectr. Electr. Insul. 2014, 21, 53-63. [CrossRef]

10. Dissado, L.A. Understanding electrical trees in solids: from experiment to theory. IEEE Trans. Dielectr. Electr. Insul. 2002, 9, 483-497. [CrossRef]

11. Jarvid, E.M.; Johansson, A.B.; Blennow, J.H.M.; Andersson, M.R.; Gubanski, S.M. Evaluation of the performance of several object types for electrical treeing experiments. IEEE Trans. Dielectr. Electr. Insul. 2013, 20, 1712-1719. [CrossRef]

12. Iddrissu, I.; Zheng, H.; Rowland, S.M. Electrical tree growth in epoxy resin under DC voltages. In Proceedings of the IEEE International Conference on Dielectrics (ICD), Montpellier, France, 3-7 July 2016; pp. 820-823. 
13. Du, B.X.; Su, J.G.; Han, T. Electrical tree characteristics of XLPE under repetitive pulse voltage in low temperature. IEEE Trans. Dielectr. Electr. Insul. 2015, 22, 1801-1808. [CrossRef]

14. Du, B.X.; Zhu, L.W. Effects of magnetic field on electrical tree growth in silicone rubber under repetitive pulse voltage. IEEE Trans. Dielectr. Electr. Insul. 2015, 22, 1785-1792. [CrossRef]

15. ASTM International. Standard Test Method for Evaluation of Resistance to Electrical Breakdown by Treeing in Solid Dielectric Materials Using Diverging Fields; ASTM D3756-97; ASTM International: West Conshohocken, PA, USA, 2010.

16. Qi, X.; Boggs, S. Thermal and mechanical properties of EPR and XLPE cable compounds. IEEE Electr. Insul. Mag. 2006, 22, 19-24. [CrossRef]

17. Abramowitz, M.; Davidson, M.W. Microscope Objectives: Immersion Media. Olympus Microscopy Resource Center. Available online: http:/ / www.olympusmicro.com/primer/anatomy/immersion.html (accessed on 10 January 2017).

18. Babadi, S.; Ramirez-Iniguez, R.; Boutaleb, T.; Mallick, T. Performance comparison of a freeform lens and a CDTIRO when combined with an LED. IEEE Photonics J. 2017, 9, 1-8. [CrossRef]

19. Liu, Y.; Cao, X. Electrical tree initiation in XLPE cable insulation by application of DC and impulse voltage. IEEE Trans. Dielectr. Electr. Insul. 2013, 20, 1691-1698. [CrossRef]

20. Griac, J.; Adamec, V.; Calderwood, J.H. On the comparability of single and double needle tests for treeing resistance. IEEE Trans. Electr. Insul. 1982, 17, 356-358. [CrossRef]

21. McMahon, E.J. A tutorial on treeing. IEEE Trans. Electr. Insul. 1978, 13, 277-288. [CrossRef]

22. Wang, Y.; Li, G.; Wu, J.; Yin, Y. Effect of temperature on space charge detrapping and periodic grounded DC tree in cross-linked polyethylene. IEEE Trans. Dielectr. Electr. Insul. 2016, 23, 3704-3711. [CrossRef]

23. Li, Z.; Liu, R.; Wang, H.; Liu, W. Space charges and initiation of electrical trees. IEEE Trans. Electr. Insul. 1989, 24, 83-89. [CrossRef]

24. Mammeri, M.; Laurent, C.; Nedjar, M. Dynamics of voltage polarity reversal as the controlling factor in space-charge induced breakdown of insulating polymers. IEEE Trans. Dielectr. Electr. Insul. 1997, 4, 44-51. [CrossRef]

25. Champion, J.V.; Dodd, S.J.; Zhao, Y.; Vaughan, A.S.; Brown, M.; Davies, A.E.; Sutton, S.J.; Swingler, S.G. Morphology and the growth of electrical trees in a propylene/ethylene copolymer. IEEE Trans. Dielectr. Electr. Insul. 2001, 8, 284-292. [CrossRef]

26. Kudo, K. Fractal analysis of electrical trees. IEEE Trans. Dielectr. Electr. Insul. 1998, 5, 713-727. [CrossRef]

27. Chen, X.; Xu, Y.; Cao, X.; Dodd, S.J.; Dissado, L.A. Effect of tree channel conductivity on electrical tree shape and breakdown in XLPE cable insulation samples. IEEE Trans. Dielectr. Electr. Insul. 2011, 18, 847-860. [CrossRef]

28. Ren, M.; Zhou, J.; Song, B.; Zhang, C.; Dong, M.; Albarracín, R. Towards optical partial discharge detection with micro silicon photomultipliers. Sensors 2017, 17, 2595. [CrossRef] [PubMed] 